\title{
El concepto de esfera pública en el ámbito de las relaciones públicas
}

\author{
María Isabel Míguez González \\ Universidad de Vigo (España)
}

Recibido: $29 / 11 / 2010$

Aceptado: 20/1/2011

\begin{abstract}
Resumen: El texto profundiza en el concepto y las características de la esfera pública y en su vínculo con las relaciones públicas. Se concluye que este constructo ayuda a explorar la función social de las relaciones públicas y permite complementar la visión organizacional de la disciplina. Además, se apunta que, como consecuencia de la implantación de las nuevas tecnologías, las relaciones públicas deben ayudar a gestionar la identidad organizacional en un nuevo universo comunicativo que amplía las posibilidades de participación de los individuos en la esfera pública.
\end{abstract}

Palabras clave: Relaciones públicas / esfera pública / visión social / participación

\section{The concept of public sphere in the field of public relations}

Summary: This text studies the concept of public sphere, its characteristics and its relation with public relations. We have concluded that this term could be helpful to explore the social function of public relations and to complete the organizational point of view of the discipline. Furthermore, we suggest that, as a consequence of the use of new technologies, public relations should help to manage the organizational identity in a communicative context that gives to the individuals more possibilities of participation in the public sphere.

Keywords: Public relations / public sphere / social function / participation 


\section{Introducción}

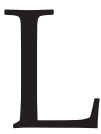

a mayoría de la doctrina sobre las relaciones públicas está basada en una visión directiva de la actividad, fundamentada en la teoría de sistemas y centrada en atención a los públicos como elemento crucial de acción. Sin embargo, desde hace años, las relaciones públicas son exploradas desde otras perspectivas (crítica, retórica, posmodernismo, etcétera) y se han introducido nuevos conceptos que comienzan a ser de uso habitual en los trabajos de muchos teóricos. Uno de estos conceptos es el de esfera pública, que se ha comenzado a aplicar en el ámbito de las relaciones públicas hace apenas dos décadas (especialmente en el ámbito europeo).

En la actualidad es posible encontrar bastantes trabajos que tratan sobre relaciones públicas y que asumen la esfera pública como elemento crucial dentro de su planteamiento, o como constructo de especial interés dentro de la disciplina.

En relación con los públicos, por ejemplo, Leitch y Neilson (2001: 127138) hablan de multiplicidad, refiriéndose a las diversas posiciones que pueden ocupar los individuos de distintos públicos en múltiples lugares de la esfera pública. Chay-Nemeth (2001: 127-162), por su parte, entiende los públicos como espacios de discurso y plantea la necesidad de dar a los públicos pasivos más oportunidades de participación en la esfera pública.
Atendiendo a la cuestión de la comunicación simétrica, Hiebert (2004: 1-9) plantea cómo las nuevas tecnologías contribuyen a la democracia mediante la restauración del diálogo y la comunicación participativa en la esfera pública. Igualmente, Roper (2005: 69-86) cuestiona la ética del proceso de comunicación simétrica porque contribuye a mantener la hegemonía y el poder de las organizaciones, en lugar de fomentar la negociación colaborativa necesaria para el correcto desarrollo de la esfera pública.

Por último, abordando aspectos de la estructura de las relaciones públicas, Wehmeier (2006: 213-220) habla de cómo las organizaciones pueden caracterizarse como un mito para alcanzar la legitimidad social en el contexto de una esfera pública compleja y de qué modo las relaciones públicas pueden desarrollarse en este contexto. Por su parte, Falkheimer (2007: 287293) aplica a la disciplina la teoría de la estructuración de Giddens, en la que la esfera pública desempeña un papel fundamental.

En este contexto, puede resultar oportuno profundizar en el tratamiento que se ha dado al constructo de esfera pública en el ámbito de las relaciones públicas $\mathrm{y}$, por este motivo, el objetivo de este estudio es realizar una revisión sobre la incorporación de este concepto en este campo, valorando su utilidad para el desarrollo de la disciplina. Para ello, se ha realizado una revisión bibliográfica y se han 
empleado, fundamentalmente, los textos de tres autoras: Sholar (1994: 77-92), Jensen (2001: 133-147) y Raupp (2004: 309-316). El trabajo de Sholar es uno de los pioneros en el tratamiento del concepto de esfera pública dentro del ámbito de las relaciones públicas, mientras que las aportaciones de Jensen y Raupp son mucho más recientes, por lo que incorporan y conjugan ideas desarrolladas de forma aislada por otros autores.

\section{Esfera pública: Concepto y características}

El concepto de esfera pública propuesto por Habermas (1981) se refiere a la suma de espacios públicos en los que todos los ciudadanos pueden entrar libremente para contribuir al debate público. Asen (2003: 177) define la esfera pública como foros discursivos parcialmente superpuestos en los que diversos ciudadanos interactúan para abordar preocupaciones compartidas. Y Raupp (2004: 310-311) habla de la esfera pública como un espacio de comunicación abierto a todos y con ciertas reglas y ciertas estructuras. Según Roper (2005: 71), esta es la esfera de la sociedad civil y se distingue por el discurso abierto entre los individuos que permite la formación racional de la opinión pública; la base necesaria para la acción comunicativa en esta esfera es que el diálogo es libre de intentos estratégicos y la opinión pública resultante es negociada en colaboración.
Sin embargo, las características que se asocian a la esfera pública en sus primeras fases son bastante más limitadas. Según Watson y Hill (2003: 238), la esfera pública surge a mediados del siglo XVII como lugar de debate e intercambio comunicativo; aunque, como añade Jensen (2001: 135), en su forma original se limita a una asamblea de ciudadanos en una localización concreta.

El nacimiento de la esfera pública se produce, según Jensen (2001: 134), en el marco de las democracias liberales, cuando se comienza a comprender que el poder autoritario debe desaparecer para dar paso a gobiernos que tienen que ser controlados por el público. Este control se ejerce mediante la participación de los ciudadanos en el discurso público, gracias a la existencia de nuevos derechos como la libertad de reunión, la libertad de expresión o la libertad de prensa. Pero esto no significa que la esfera pública haya estado siempre abierta a la participación de todo el mundo; de hecho, según Sholar (1994: 78), inicialmente presenta un carácter eminentemente burgués y excluye a las mujeres y las clases trabajadoras.

Estos aspectos limitadores de las primeras esferas públicas han dejado de tener vigencia a medida que se han ido perfeccionando los sistemas democráticos, aunque la evolución histórica también ha supuesto cambios negativos. Así, según Watson y Hill (2003: 238), desde mediados del siglo 
XIX la esfera pública ha estado dominada por el Estado y por los intereses económicos, por lo que ha perdido su finalidad original de funcionar como una zona neutral de discurso público. De todas formas, según los autores, las nuevas tecnologías ofrecen la posibilidad de recuperar este concepto, ya que internet puede actuar hoy como una extensión de la esfera pública, al ser un espacio libre para el discurso.

A pesar de las variaciones en el concepto y de los cambios de escenario, sigue existiendo un amplio consenso en torno a los fundamentos de la idea de esfera pública: para que pueda alcanzarse una comunicación democrática ha de sustituirse el poder autoritario por el razonamiento discursivo, esencia de la esfera pública (Sholar 1994: 78), y este razonamiento discursivo de carácter público sigue dependiendo en la actualidad de la existencia de la libertad de asamblea, de expresión y de asociación (Jensen 2001: 135).

Según Sholar (1994: 78-80), gracias, precisamente, a elementos como la libertad de asamblea y de información o el derecho al voto, la esfera pública está protegida por la ley y solo un sistema democrático en el que todos los ciudadanos tengan las mismas posibilidades de acceder a las fuentes de información y las mismas oportunidades para participar en los debates de los que surgen las decisiones políticas proporciona un marco óptimo para el desarrollo de la esfera pública en su estado ideal. Estas normas que afectan la esfera pública constituyen, según Raupp (2004: 311), su estructura formal; dentro de ella, los sujetos que se comunican intentarán atraer la atención de los demás sobre ciertos mensajes y ciertos temas y su éxito en esta tarea dependerá de los recursos que sean capaces de invertir.

Por otra parte, se entiende que todos los actores que desempeñan un papel en la esfera pública (concebida de forma ideal) deben ser iguales, sin que unos tengan más poder que otros. Por tanto, la esfera pública es una estructura en la que cada ciudadano ejerce su derecho al debate público, siguiendo unas reglas similares para todos y buscando unas metas que no deben centrarse en intereses privados, sino en preocupaciones públicas, tal y como afirma Sholar (1994: 78-80).

El número de personas potencialmente involucradas en los procesos comunicativos de la esfera pública es variable. En este sentido, según Raupp (2004: 311), se podrían distinguir tres niveles: a) el nivel de los encuentros públicos, en el que los individuos entran en contacto directo; b) el de las reuniones públicas, un poco más amplio (como un discurso de una campaña electoral, por ejemplo); o c) el nivel de los mass media, la forma más amplia de la esfera pública.

Esta clasificación contempla solo procesos temporalmente limitados. Pero, según Sholar (1994: 80-82), los procesos de la esfera pública también 
pueden situarse, de modo más o menos permanente, en ciertas organizaciones, como las asociaciones culturales, religiosas, políticas, cívicas o comunitarias. Sin embargo, delimitar el modo en que pueden formar parte de los procesos de la esfera no resulta sencillo, ya que estas organizaciones han crecido de un modo vertiginoso en los últimos años y se han vuelto más complejas y burocráticas, lo que podría limitar su funcionamiento autónomo, perjudicando su papel como agentes independientes en la esfera.

En cuanto al papel que desempeñan los medios de comunicación en la esfera pública, Sholar (1994: 82) afirma que los medios creados y gestionados por diferentes grupos asociativos para sus fines, como periódicos o revistas propios, podrían ser considerados como parte de los procesos de la esfera pública. Sin embargo, no ocurre lo mismo con los medios comerciales, que presentan reglas internas que limitan las posibilidades de discurso público (sus empleados y periodistas filtran los intentos de los individuos de comunicarse en la esfera pública) y a menudo actúan como espacio para la publicity relacionada con intereses económicos y estatales.

Jensen (2001: 135-136) añade que, aunque los medios no son la esfera pública, esta sí depende de su libertad y debe prevenir su monopolio. Asimismo, la esfera pública no supone un conjunto de valores y opiniones co- munes, pero puede influir en las opiniones que se forman en la sociedad $y$, a pesar de que no necesariamente genera acuerdos, puede influir en las decisiones que toman los individuos, las instituciones y los gobiernos. En síntesis, el discurso de la esfera pública representa un modo civilizado de manifestar el desacuerdo en torno a temas de preocupación común. Por eso, no representa una posición general ni consensuada, sino que supone "un complejo dinámico de diversas posiciones conflictivas" (Jensen 2001: 136).

De todas formas, los intereses económicos y políticos degradan a veces la esencia de la esfera pública. Esto se manifiesta, por ejemplo, en que los medios comerciales, como instituciones que facilitan el intercambio de información y opinión entre el público, han comenzado a ser vistos, según Sholar (1994: 83-84), como parte de la esfera pública. Pero acceder a estos medios supone un coste elevado, lo que significa que la posibilidad de participar en los procesos democráticos de comunicación se ha convertido en una cuestión de poder económico. Así, la concentración de poder en la industria de la comunicación hace, según Sholar, que los posibles oradores del discurso público se reduzcan cada vez más.

Pese a esto, Sholar considera que las degradaciones que se producen en los procesos de la esfera pública no deben enturbiar la comprensión de la esfera, como constructo normativo, en su estado ideal. 


\section{La incorporación de la noción de esfera pública a las relaciones públicas}

Aunque cada vez son más frecuentes los textos sobre relaciones públicas que hablan de la esfera pública, Raupp (2004: 309-310) opina que aún no se ha tratado este asunto de un modo consistente y para hacerlo se debe partir del análisis del adjetivo "público", fundamento tanto del concepto "públicos", como sustantivo plural, como del concepto de esfera pública.

Este adjetivo designa, originariamente, aquello que afecta a todos o que es accesible a todos y con el nacimiento de las naciones-Estado pasa a ser sinónimo de lo que es propiedad de ese Estado o lo que está regulado por él. Según Raupp, la idea de los públicos, entendidos como grupos de personas que están en el entorno de una organización, es el eje de las corrientes de las relaciones públicas que centran su estudio en el nivel organizacional y que se desarrollan, sobre todo, en Estados Unidos. Sin embargo, parte de la teoría europea de las relaciones públicas trabaja especialmente sobre el nivel social de las relaciones públicas; y en este contexto, no deben ser los públicos el elemento fundamental, sino la esfera pública, entendida, al contrario que la última acepción del adjetivo público, como aquello que pertenece a los ciudadanos o que no está regulado por el Estado ni es de su propiedad.

El origen del interés de los teóricos por la idea de esfera pública deriva de las limitaciones del concepto de público. Para muchos autores, la idea del público en general es una imposibilidad lógica y aconsejan identificar y diferenciar públicos que tengan consecuencias similares para la organización; pero, para Jensen (2001: 133), este punto de vista hace que los públicos se conviertan en algo parecido a los segmentos de mercado del marketing, y cree que con ello se pierde la oportunidad de analizar las relaciones públicas en relación con temas y valores de relevancia pública. Raupp (2004: 314), más benevolente que Jensen en su crítica a las propuestas tradicionales, opina que las teorías situacionales de identificación de los públicos, concebidos como audiencias fragmentadas, van más allá de los sencillos modelos de marketing y se centran con interés en los procesos de comunicación; sin embargo, coincide con Jensen en que esta consideración de los públicos como los elementos más relevantes para la práctica de las relaciones públicas impide comprender los procesos de formación de la opinión en sociedades mediáticas, que no se limitan a estos grupos sociales.

En síntesis, la atención exclusiva de las relaciones públicas por los públicos específicos hace que se pierda la consideración del nivel social de la disciplina y recurrir al concepto de esfera pública es, precisamente, una de las formas de entender con mayor claridad este papel social de las relaciones públicas. 
De todas formas, esto no implica que los públicos estratégicos dejen de ser importantes. Según Raupp (2004: 310-315), ni la perspectiva de los públicos ni la de la esfera pública son completas por sí mismas, ya que la única forma de comprender los vínculos entre los procesos de comunicación que se dan en distintos ámbitos es conjugar ambos niveles, por lo que es necesaria una perspectiva integradora que una las dos visiones. En este sentido, la autora plantea que las perspectivas de las relaciones públicas basadas en el concepto de esfera pública tienden a entenderlo como un factor constituyente de la democracia o como un elemento destinado a construir realidades deseables. Pero, tratando de aproximar esta visión a la visión de los públicos, la autora considera más adecuado entender la esfera pública como un espacio de comunicación que permite atraer la atención de los individuos hacia ciertas cuestiones y mensajes en diferentes áreas de la comunicación pública para influir en los procesos de discusión pública; y, en este contexto, los públicos son actores sociales que, dentro de la esfera pública, se agrupan en torno a temas específicos y deben ser identificados por la organización para establecer con ellos una comunicación estratégica.

Los conceptos de esfera pública y de públicos son, por tanto, interdependientes y son tanto los requisitos previos como el producto de los procesos de comunicación a los que dan lugar las relaciones públicas en la es- fera pública. En síntesis, según Raupp, las relaciones públicas cumplen dos funciones: una función social, al proporcionar temas para la comunicación en la esfera pública, y una función organizacional, al gestionar las relaciones de comunicación con los públicos que se agrupan en torno a estos temas. Esta última visión ya ha sido explorada en numerosas ocasiones; pero sobre la función social de las relaciones públicas se ha trabajado menos $\mathrm{y}$, para ello, es imprescindible determinar el papel de las relaciones públicas en la esfera pública.

\section{El papel de las relaciones públicas en la esfera pública}

Citando a Danielian (1939), Sholar (1994: 84) afirma que todas las organizaciones desean alcanzar una representación de sí mismas en la esfera pública. Pero, cuando estas organizaciones tienen intereses económicos o buscan, únicamente, la defensa de sus intereses privados, se genera un conflicto, ya que la esfera pública está reservada para la discusión y debate de intereses públicos. Por eso, para ocupar un lugar en la esfera, las organizaciones capitalistas económicas no solo deben legitimar en ella sus prácticas -lo que se correspondería, en cierto modo, con la demostración de su responsabilidad social-, sino también su propia existencia.

Jensen (2001: 136-137) corrobora este planteamiento cuando diferencia 
tres posibles funciones de la esfera pública. Según la autora, existiría una esfera literaria, relativa a los procesos por los que diversos agentes hacen públicos sus textos en la esfera para reflejar su persona, generando un proceso continuo de reflexión sobre las experiencias personales que permite a los individuos y las organizaciones desarrollar su identidad y su concepto sobre los otros.

En segundo lugar, la esfera pública presenta una función política, referida a los discursos en los que se identifican y se exponen problemas sociales de interés común que pueden tener una solución por vía gubernamental o reglamentaria; es decir, se plantean críticas y peticiones dirigidas a políticos y gobiernos para que actúen, dando, al mismo tiempo, cierta legitimidad a estas actuaciones.

Por último, en los años recientes estaría emergiendo una tercera esfera en la que se discute la identidad y la legitimidad de organizaciones que pueden causar problemas que afectan a todos pero para los que no existe una solución por vía política o gubernamental; $y$, desde nuestro punto de vista, este es el nivel al que se refiere Sholar (1994: 86) cuando apunta que las organizaciones deben legitimar en la esfera pública sus acciones y su propia existencia.

En este sentido, el papel de las relaciones públicas ha de ser precisamente, según Sholar: crear a través de la publicity representaciones de la organización en la esfera pública; construir públicos y asociar los intereses privados de la organización con los intereses de estos públicos representativos; construir ideologías o planteamientos de buena voluntad sobre cómo debería funcionar el mundo, y financiar el discurso de otras organizaciones que, por no presentar intereses económicos, sí están legitimadas dentro de la esfera pública.

Para realizar estas y otras actuaciones, los profesionales deben tener en cuenta, según Raupp (2004: 311-321), el nivel en el que actúan, porque cada uno presenta diferentes reglas relevantes para la práctica de las relaciones públicas. Los encuentros directos implican una interacción también directa de los individuos, cara a cara o a través de medios que simulan esta situación; en estos encuentros, cada individuo recibe una respuesta inmediata a su intervención, por lo que puede valorar en el momento los efectos que ha conseguido con ella.

En las reuniones públicas se distribuyen con más claridad los roles de orador y oyente, por lo que la interacción -o la posibilidad de intervención de la audiencia- es limitada y el orador debe elaborar más la comunicación, porque se dirige a muchas personas simultáneamente. Por último, en el nivel de los medios, el feedback se produce únicamente de modo excepcional y los roles de comunicadores (organizaciones, gobiernos, etcétera), intermediarios (periodistas) y receptores no se pueden modificar. 
No conviene olvidar, de todos modos, que los niveles de actuación en la esfera pública son cada vez más confusos, como consecuencia de la incorporación de las nuevas tecnologías. Tal y como apuntan Cozier y Witmer (2001: 615-623), la emergencia de comunidades virtuales, a través de diferentes formas de comunicación en línea, ha generado un nuevo entorno para las organizaciones. Aplicando esto a los niveles mencionados por Raupp, cabría apuntar que se han multiplicado los encuentros directos simulados, que las reuniones públicas ya no tienen por qué ser presenciales y permiten más fácilmente el intercambio de roles y que, gracias a la presencia de los grandes medios comerciales en internet, el feedback ya no es tan excepcional. Además, los nuevos discursos públicos a través de la red no están limitados temporal ni espacialmente, lo que hace que las organizaciones deban replantearse sus modos de relación, en un entorno que multiplica las posibilidades de intervención de los individuos en la esfera pública.

De todas formas, no todas las organizaciones se relacionan de igual modo en la esfera pública ni los profesionales de las relaciones públicas realizan en ellas las mismas funciones. Así, Jensen (2001: 133-147) plantea que existen tres tipos de compañías, con diferencias en su participación en la esfera y en su actividad de relaciones públicas.

En primer lugar, las compañías económicamente exitosas pero social- mente inocentes son empresas propias de las economías liberales de mercado. Se orientan al mercado, únicamente se preocupan por las preferencias de sus clientes, proveedores, inversores, etcétera y no creen que sus stakeholders estén preocupados por sus valores sociales. Así, no se relacionan con la esfera pública, no incluyen en su comunicación aspectos relacionados con el bien común, no consideran que las relaciones públicas sean relevantes y solo realizan actividades de marketing. Además, su posición está avalada por los discursos políticos, que no confían en que la compañía pueda ser responsable por sí misma.

En segundo lugar, las compañías económicamente exitosas y legales se dan en contextos en los que se asume que los gobiernos han de ejercer un cierto intervencionismo para suplir las deficiencias del mercado en la distribución equitativa de los bienes en la sociedad. Estas organizaciones se relacionan con la esfera pública política, porque deben seguir leyes y normativas relacionadas, por ejemplo, con sus efectos sobre la salud o el medio ambiente. Dado que tratan de asegurar un marco legal para sus actividades, sus relaciones públicas tienen la función de analizar las tendencias del debate público para pronosticar posibles regulaciones que puedan restringir o apoyar el negocio. Además, sus relaciones públicas también pueden asumir una función proactiva, tomando parte de estos discursos (activida- 
des de lobbying y de asuntos públicos) para asegurar que la perspectiva de la entidad se tiene en cuenta o para buscar soluciones que puedan mejorar el marco legal de la compañía. En ocasiones, las relaciones públicas también pueden asumir actividades clandestinas para suplir la falta de credibilidad que estas compañías suelen tener al defender sus puntos de vista sobre temas de interés común en los discursos políticos públicos. Pero estas acciones pueden resultar contraproducentes, porque si los medios de comunicación las descubren, la compañía perderá de forma definitiva su credibilidad como agente de la esfera pública.

Por último, las compañías económicamente exitosas, legales y responsables, se preocupan de las consecuencias de su actividad más allá de lo legal y tratan de responder a las demandas públicas. Su aparición se debe, en parte, a la creencia de que el gobierno no puede resolver todos los problemas ni regular de forma exhaustiva cada negocio, por lo que se empieza a exigir responsabilidad a las propias organizaciones, que han de ser capaces, además, de demostrar su razón de ser y su legitimidad. Es decir, han de moverse en la tercera esfera pública, que tiene muchas similitudes con la esfera pública literaria, ya que los discursos de las organizaciones van hacia la esfera pública y de esta vuelven hacia la organización para que esta compruebe su identidad. Para ello, la organización se involucra en muchos discursos públicos, tiene muchos stakeholders $\mathrm{y}$ sus funciones de relaciones públicas, reactivas y proactivas, son muy amplias. Sin embargo, sus modelos se siguen basando en la centralidad de la organización frente a los stakeholders, lo que niega la posibilidad de procesos discursivos bien desarrollados.

Por eso, según Jensen, es necesario generar teorías de las relaciones públicas en las que se analicen en profundidad las características de los procesos de la esfera pública y en los que la organización no sea el centro de todos los procesos.

\section{Reflexiones finales}

El concepto de esfera pública no es, todavía, un constructo esencial en los modelos de investigación de las relaciones públicas. Sin embargo, poco a poco va adquiriendo peso, los estudios que se ocupan de él comienzan a ser más abundantes y cada vez son más los teóricos no europeos dedicados al tema.

Las limitaciones del concepto de público y la convicción de que las relaciones públicas deben cumplir una función social son los desencadenantes de la introducción de la idea de esfera pública en este ámbito. Sus defensores insisten en la necesidad de las organizaciones de legitimar su identidad en esta esfera y consideran excesivamente estrecha la visión organizacional de las relaciones públicas derivada de los modelos que sitúan a los públicos específicos como únicos interlocutores de la organización. 
De todos modos, estos autores no rechazan la idea de que una organización pueda dirigirse a públicos concretos, sino que plantean, simplemente, la necesidad de que la actividad de las relaciones públicas se extienda a un ámbito mayor, adquiriendo un compromiso ético con el conjunto de la sociedad, al tiempo que reivindican la capacidad de los propios integrantes de la sociedad para ser, a su vez, partícipes de los procesos comunicativos de la esfera pública. Así, lo que se formula, en realidad, es que la organización debe atender dos dimensiones de la comunicación: una estructural, basada en la comunicación en la esfera pública, como lugar en el cual alcanzar una representación y legitimar una identidad; y una encaminada a la acción, basada en la comunicación con los públicos, que, como actores sociales dentro de la esfera pública, se agrupan en torno a temas específicos y deben ser identificados por la organización.

En cierto modo, se recupera la idea de que las organizaciones, además de ocuparse de sus públicos específicos, deben comunicarse con el público genérico, pero este concepto ya anticuado y descartado se sustituye por el de esfera pública y por la idea, más elaborada, de que las compañías han de intervenir en el espacio público en el que se producen las interacciones comunicativas para ganar legitimidad y presencia social.

Los condicionantes para la participación de las organizaciones en la esfera pública son cada vez más im- portantes, porque se apela a la responsabilidad como elemento esencial para la legitimación de las entidades como actores en el discurso público. Y, al mismo tiempo, las posibilidades de los individuos de participar en la esfera y contribuir a la legitimación o deslegitimación de las organizaciones es cada vez más alta, porque, gracias a las nuevas tecnologías, se han desarrollado múltiples mecanismos que permiten a los individuos opinar, interactuar en la esfera e influir en sus procesos.

En este nuevo marco, la consideración del conjunto de la esfera pública es fundamental para las organizaciones y para los profesionales de las relaciones públicas, cuyos nuevos retos no se encuentran tanto en el trato estratégico habitual con sus públicos específicos, como en la gestión de la identidad organizacional en un universo comunicativo más amplio, cambiante y participativo.

\section{Bibliografía}

Asen, Robert (2003). “The multiple Mr. Dewey: multiple publics and permeable borders in John Dewey's Theory of the Public Sphere". Argumentation and Advocacy. Vol. 39. American Forensic Association.

Chay-Nemeth, Constance (2001). "Revisiting publics: a critical archaeology of publics in the Thai HIV/AIDS issue". Journal of Public Relations Research. Vol. 13, núm. 2. Londres: Taylor \& Francis Group. 
Cozier, Zoraida R. y Diane F. Witmer (2001). "The development for a structuration analysis of new publics in a electronic environment", en Heath, Robert L. (ed.). Handbook of public relations. Thousand Oaks: Sage.

Danielian, Noorbar R. (1939). ATET: The story of industrial conquest. Nueva York: Vanguard Press.

FALKHeIMER, Jesper (2007). "Anthony Giddens and public relations: A third way perspective". Public Relations Review. Vol. 33. Nueva York: Elsevier.

Habermas, Jurgen 1981. Historia y crítica de la opinión pública: La transformación estructural de la vida pública. Barcelona: Gustavo Gili. [Versión original en alemán de 1962.]

Hiebert, Ray E. (2004). “Commentary: New technologies, public relations, and democracy". Public Relations Review. Vol. 31. Nueva York: Elsevier.

Jensen, Inger (2001). "Public relations and emerging functions of the public sphere: an analytical framework". Journal of Communication Management. Vol. 6. Bingley: Emerald Group.

Leitch, Shirley y David Neilson (2001). "Bringing publics into public rela- tions: New theoretical frameworks for practice", en Нвath, Robert L. (ed.). Handbook of Public Relations. Thousand Oaks: Sage.

Raupp, Juliana (2004). "The public sphere as a central concept of public relations", en Ruler, Betteke van y Dejan V̌̆Rcic (eds.). Public relations and communication management in Europe. Nueva York: Mouton de Gruyter.

Roper, Juliete (2005). “Symmetrical communication: Excellent Public Re-lations or a strategy for hegemony?". Journal of Public Relations Research. Vol. 17, núm. 1. Londres: Taylor \& Francis Group.

Sholar, Sylvia (1994). "E. Habermas, Marx and Gramsci: investigating the public sphere in organizational communication and public relation courses". Journal of Communication Inquiry. Vol. 18, núm. 20. Thousand Oaks: Sage Publications.

Watson, James y Anne Hill (2003). Dictionary of media and communication studies. 6. ${ }^{a}$ edición. Londres: Arnold.

Wehmeier, Stephan 2006. "Dancers in the dark: The myth of rationality in public relations". Public Relations Review. Vol. 32. Nueva York: Elsevier. 